\title{
Bilateral hip osteonecrosis: influence of hip size on outcome
}

Ph Hernigou, J C Lambotte

\begin{abstract}
Objective-To measure the volume of osteonecrosis in 30 patients with disease in both hips (one side with collapse and the other without at Ficat stage I).

Method-The volume of the osteonecrosis was measured by magnetic resonance imaging.

Results-The progression to collapse was influenced by the size of the lesion in each patient. The first collapsed hip was that with the largest volume of osteonecrosis. Conclusion-The volume of the osteonecrotic lesion on the second hip (stage I without collapse) is a good predictor of the time to collapse of this second hip.

(Ann Rheum Dis 2000;59:817-821)
\end{abstract}

The treatment of osteonecrosis of the femoral head remains a challenging problem because both hips are commonly affected and, often, patients have systemic illness with persisting underlying disease or risk factors. One treatment option for stage I (the shape of the joint is unaltered, the $x$ ray findings are normal, and the diagnosis is made with magnetic resonance imaging (MRI) only) and for stage II (the shape of the joint is still preserved and the articular surface is intact, but the diagnosis can be done with an $x$ ray examination) is conservative management, usually core decompression. Once collapse has occurred (stage III), arthroplasty is often indicated as the disease has reached the stage of no return because the joint surface is altered as a result of collapse in the necrotic region.

Intuitive reasoning and observation of many femoral head osteonecroses suggest that the outcome of femoral head osteonecrosis, and, particularly, the evolution to collapse, is influenced by the size of the lesion and also by the time from the diagnosis. ${ }^{1-3}$ Many methods have been used to estimate or measure the size of the lesion by radiography or by MRI. ${ }^{45}$ These studies have indicated that the extent of the necrotic lesion affects the outcome. As the patients and the causes of necrosis differ and because the time of onset of osteonecrosis was unknown, studies ${ }^{67}$ were done with survival curves using a Kaplan-Meier method. Furthermore, exact volumetric analysis was not used in the following studies..$^{8-10}$

The purpose of this report is to study the influence of the size of the lesion on collapse in a group of patientswith bilateral osteonecrosis, one side with collapse and the other side without, using volumetric analysis of the lesion.

\section{Materials and methods \\ PATIENTS}

In this study we evaluated 30 patients with bilateral hip osteonecrosis (one side with collapse and the other without). Patients were referred to the orthopaedic surgery unit because they were sufficiently symptomatic on one side for surgery to be warranted. On this side $x$ ray findings showed collapse and the hip was operated on (total hip arthroplasty). Only patients with bilateral osteonecrosis, shown by MRI, and presenting one hip with collapse and the other asymptomatic without collapse and stage I (Ficat classification) were included in the study. Hips with stage II osteonecrosis were not included as it may sometimes be difficult to differentiate between stage II and stage III even with careful screening using ascendant (30 degrees) $x$ rays, lateral $x$ rays, tomograms, or computed tomography to check the subchondral crescentic line or collapse. The authors are aware of other combinations of patients presenting with bilateral osteonecroses-for example, both sides presenting as collapsed or as non-collapsed or one side with stage II and the other side with or without collapse. However, if stage II were used this might include patients with one side in a precollapsed state which might soon become collapsed and thus not allow evaluation of the rate of progression. When these inclusion criteria were used (bilateral MRI, one hip with collapse, the other not) 30 patients were included in the study over a period of two years from among 80 patients with osteonecrosis who were seen consecutively during this time.

These osteonecroses were associated with corticosteroid treatment in 25 patients. This treatment had been given for systemic lupus erythematosus (four patients), asthma (seven), haematological malignancy (10), renal transplantation (three), and cardiac transplantation (one). Of the 25 patients receiving corticosteroids, 18 had been treated only for a short time (less than four months). This group of 18 patients was considered separately as the short exposure to corticosteroids allowed a better approximation of the time of onset of the osteonecrosis. For three patients the risk factor was admitted alcohol abuse, and two other patients had sickle cell disease.

The collapsed hip had significant dysfunction and was treated with hip arthroplasty; the other hip (stage I) had no treatment. We had no histological confirmation for stage I hips, but the diagnosis of stage I osteonecrosis is supported by the fact that all these hips had a typical band pattern; ${ }^{112}$ histology was confirmed for the other collapsed hip. All these hips had MRI performed at the time of the first
Accepted for publication 28 March 2000 
evaluation before surgery with a $1.5 \mathrm{~T}$ superconducting unit; serial coronal sections through the hip were obtained with $T_{1}$ weighted spin echo sequences. All these hips also had standard radiographs (frontal, lateral, and ascendant 30 degrees) to look for collapse. The non-collapsed hip was followed up every three months (by radiological examination) to look for the first indication of collapse and to discover how the collapse was related to the passage of time. The end point of stage I for the second hip was the date of radiological collapse and not the need for reconstructive surgery because of significant dysfunction. All the hips with stage I at the time of presentation had a second MRI scan two years later. So in this series three different groups of hips will be compared: (a) hips with collapse at the first MRI scan (MRI1) stage III hips; (b) hips without collapse at the first MRI scan (MRI1) stage I hips; hips with collapse at the second MRI scan (MRI2) stage III hips.

MEASUREMENT OF THE VOLUME OF THE OSTEONECROSIS WITH MRI CORONAL SLICES Probably, in the next few years, automatic volumetric analysis with $3 \mathrm{D}$ software packages $^{13}$ will become commonplace. We measured the volume of the necrosis using contiguous MRI scans which included the whole of the femoral head. ${ }^{2}$ The thickness of each slice was always $5 \mathrm{~mm}$. For each coronal slice (mean 9.4 slices per hip), the sector of the necrosis was considered as the sector demarcated by the serpiginous band-like hypointense line. The surface of each sector of osteonecrosis was calculated on each slice. The volume of the necrosis was obtained by multiplying the sum of all the elementary surfaces by their thickness.

In practice, the surface of each cut was determined with a grid. Each line being $1 \mathrm{~mm}$ from the next. The surface was calculated by counting the number of unit squares composing the surface of the osteonecrosis on each cut (fig 1). To assess the validity of the method, the volume of osteonecroses in 30 hips selected at random was evaluated independently by the two authors. Each observer measured each osteonecrosis twice, with an interval of at least 15 days between the first and second readings. The variability between the observers was measured by defining a tolerance interval using an analysis of variance; it was estimated as 0.25 $\mathrm{cm}^{3}$ (95\% CI -2.50 to 3.00 ). The mean difference between the first and second observation in each observer was estimated as $0.05 \mathrm{~cm}^{3}$ $(95 \%$ CI -2.65 to 2.75$)$. We therefore proceeded on the assumption that both these differences were zero, and estimated the interobserver and intraobserver variance components as 0.157 and 0.065 , respectively. With this assumption, the difference between two different readings made by the same observer has a variance of $2 \times 0.157=0.314$, and therefore a standard deviation of $0.560 \mathrm{~cm}^{3}$. The difference between two different readings made by different observers has a variance of $2 \times$ $(0.157+0.065)=0.444$, giving a standard deviation of $0.666 \mathrm{~cm}^{3}$. We therefore expect

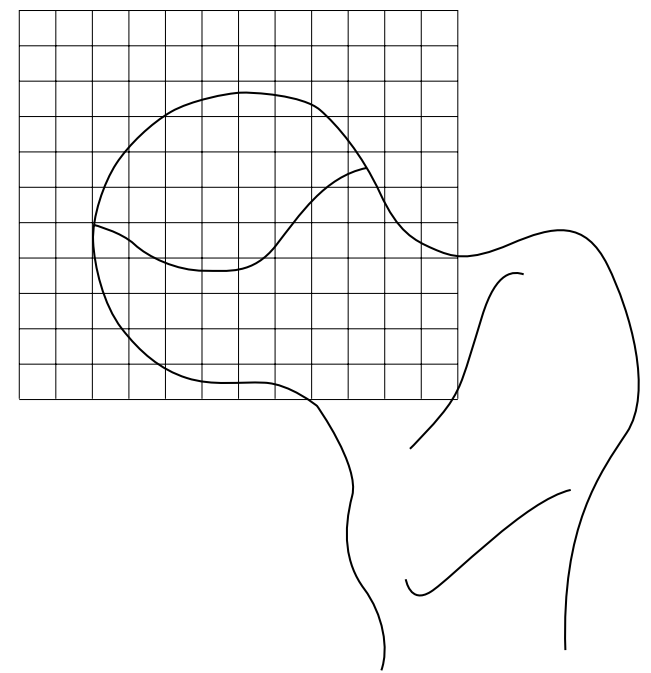

Figure 1 Diagram showing the method of determining the amount of necrosis of the femoral head by point counting with a grid on a coronal slice. On this slice the number of squares completely filled with necrosis is 15 and the number incompletely filled is 16. The number of unit squares is calculated as $15+16 / 2$ (23 unit squares). In practice, the lines were $1 \mathrm{~mm}$ apart from each other.

that $95 \%$ of intraobserver differences will have a magnitude of no more than $1.5 \mathrm{~cm}^{3}$, whereas $95 \%$ of interobserver differences will have a magnitude no more than $1.7 \mathrm{~cm}^{3}$. Thus, one can be $95 \%$ confident that $95 \%$ of the time one observer's reading will be no more than $2 \mathrm{~cm}^{3}$ more or less than the other observer's reading due to observer error alone.

The radius of the femoral head was estimated on the slice where the femoral head appeared to have the largest dimension. The radius was used to calculate the volume of the femoral head (as a sphere without cartilage) and to calculate the percentage of the femoral head affected by osteonecrosis. The radius was calculated for both hips in each patient. When the percentage of the femoral head affected was used to compare the two groups, the maximum error in measuring the volume of the femoral head was estimated to be $8 \mathrm{~cm}^{3}$. This maximum error was determined with the previously mentioned tolerance limits and with a mathematical technique. The latter calculated the variation in the volume of a sphere according to the error made in the determination of the radius; given the slice thickness of $5 \mathrm{~mm}$, the measurement centre of the femoral head may vary by $\pm 2.5 \mathrm{~mm}$. This value of $8 \mathrm{~cm}^{3}$ was used to test an unfavourable situation comparing the greatest osteonecroses (value measured- $8 \mathrm{~cm}^{3}$ ) and the smallest osteonecroses (value measured $+8 \mathrm{~cm}^{3}$ ).

\section{STATISTICAL METHODS}

A non-parametric test, the Mann-Whitney U test, was used for statistical analysis: each stage of osteonecrosis being an independent variable, the volume of the osteonecrosis and the percentage of the femoral head affected being the dependent variables. Spearman rank correlations were calculated to explore the relation between the volume and the time to collapse.

To determine the significance of the percentage of involvement as predictor of the time to 
Table 1 Volume of necrosis and percentage of the femoral head affected by osteonecrosis

\begin{tabular}{|c|c|c|c|c|}
\hline \multirow[b]{2}{*}{ Patient No } & \multicolumn{2}{|c|}{ Stage III Volume } & \multicolumn{2}{|c|}{ Stage I Volume } \\
\hline & $\mathrm{cm}^{3}$ & $\%$ & $\mathrm{~cm}^{3}$ & $\%$ \\
\hline 1 & 16.3 & 39.2 & 6.2 & 13.9 \\
\hline 2 & 10.3 & 24.8 & 8.5 & 19.1 \\
\hline 3 & 12.8 & 19.1 & 4.3 & 6.6 \\
\hline 4 & 23.9 & 57.5 & 13.8 & 21.1 \\
\hline 5 & 26.8 & 64.4 & 15.4 & 23.5 \\
\hline 6 & 26.2 & 40.1 & 16 & 29.4 \\
\hline 7 & 20.3 & 31 & 21.2 & 32.4 \\
\hline 8 & 13.3 & 20.3 & 8.8 & 17.3 \\
\hline 9 & 16.9 & 25.8 & 13.8 & 27.1 \\
\hline 10 & 12 & 18.3 & 4.6 & 7 \\
\hline 11 & 32 & 36.7 & 15.4 & 30.2 \\
\hline 12 & 18.5 & 21.2 & 3.1 & 3.8 \\
\hline 13 & 38.8 & 59.3 & 20 & 34.5 \\
\hline 14 & 21.2 & 32.4 & 17 & 26 \\
\hline 15 & 26.2 & 40.1 & 12 & 18.3 \\
\hline 16 & 43.5 & 38.5 & 5.9 & 6.8 \\
\hline 17 & 43.5 & 38.5 & 7.5 & 9.1 \\
\hline 18 & 29.6 & 51.1 & 10 & 15.4 \\
\hline 19 & 29 & 44.3 & 7.5 & 13 \\
\hline 20 & 23.2 & 40.1 & 14.6 & 37.6 \\
\hline 21 & 13 & 19.9 & 4.4 & 6 \\
\hline 22 & 27.6 & 40.7 & 12.5 & 14.4 \\
\hline 23 & 20 & 30.6 & 3.3 & 6.1 \\
\hline 24 & 6 & 9.2 & 1 & 2.4 \\
\hline 25 & 36.1 & 39.2 & 12.4 & 27.8 \\
\hline 26 & 36.7 & 56.1 & 5.5 & 11.5 \\
\hline 27 & 33.8 & 51.7 & 7.3 & 13.4 \\
\hline 28 & 25.4 & 30.8 & 8 & 14.7 \\
\hline 29 & 43.6 & 39.1 & 15.6 & 17 \\
\hline 30 & 22.5 & 34.4 & 14.6 & 22.3 \\
\hline
\end{tabular}

For all the data, $\mathrm{cm}^{3}=$ the volume of osteonecrosis in $\mathrm{cm}^{3} ; \%=$ the percentage of the femoral head affected by osteonecrosis; the volume of the femoral head is not the same on each side as determined by the radius measured on each magnetic resonance imaging scan.

collapse of the second stage I osteonecrosis, when patients already have a collapsed hip, logistic regression analysis was conducted. Kaplan-Meier analysis was used to plot survival probability with collapse as the end point of survivorship for the second hip.

\section{Results}

There was no significant difference in the mean size of osteonecrosis between the left and right sides and no significant difference in the mean size between the different causes of osteonecrosis.

At the first MRI evaluation the hips with a stage III osteonecrosis always had the largest lesions. When determined by observer I, the average volume of osteonecrosis with collapse (stage III) was 24.9 (SD 10.3) $\mathrm{cm}^{3}$. On average (table 1), the volume of osteonecrosis without collapse (stage I) was $10.3(5.34) \mathrm{cm}^{3}$. The difference between the two groups was highly significant $(p=0.0000001)$ using the MannWhitney U test. On the first MRI evaluation the percentage of the femoral head affected in the group with collapse was $36.7 \%$ (SD 13.5) and in the group without collapse $17.3 \%$ (SD 9.89). This difference was also highly significant $(p=0.0000004)$. Among the 30 hips evaluated independently by the two authors, the difference between the two readings by each observer was calculated for each hip: $95 \%$ of the differences were $2 \mathrm{~cm}^{3}$ or less (range $0-3)$. Using this value of $2 \mathrm{~cm}^{3}$ provides the most unfavourable case - that is, $\pm 2 \mathrm{~cm}^{3}$. However, when the volume of osteonecrosis at stage III (each volume $-2 \mathrm{~cm}^{3}$ ) was compared with the volume of osteonecrosis at stage I (each
Table 2 Difference in the volume between the osteonecrosis of the two hips and time before collapse for the $30 \mathrm{hips}$ evaluated as stage I at the beginning of the study. The time is the difference in months between the date of the radiograph showing collapse and the radiograph done at the first evaluation. Patients are ordered by increasing difference in volume between osteonecrosis in the two hips

\begin{tabular}{|c|c|c|}
\hline $\begin{array}{l}\text { Patient } \\
\text { No }\end{array}$ & $\begin{array}{l}\text { Difference in volume between } \\
\text { osteonecrosis in the two hips }\left(\mathrm{cm}^{3}\right)\end{array}$ & $\begin{array}{l}\text { Time in months } \\
\text { before stage III }\end{array}$ \\
\hline 7 & -0.9 & 4 \\
\hline 2 & 1.8 & 6 \\
\hline 9 & 3.1 & 6 \\
\hline 14 & 4.2 & 5 \\
\hline 8 & 4.5 & 5 \\
\hline 24 & 5 & No collapse \\
\hline 10 & 7.4 & 10 \\
\hline 30 & 7.9 & \\
\hline 3 & 8.2 & 9 \\
\hline 20 & 8.6 & 9 \\
\hline 21 & 8.6 & 8 \\
\hline 1 & 10.1 & 13 \\
\hline 4 & 10.1 & No collapse \\
\hline 6 & 10.2 & 12 \\
\hline 5 & 11.4 & 15 \\
\hline 19 & 11.5 & 12 \\
\hline 15 & 14.2 & 14 \\
\hline 22 & 15.1 & 17 \\
\hline 12 & 15.4 & No collapse \\
\hline 11 & 16.6 & 12 \\
\hline 23 & 16.7 & 18 \\
\hline 28 & 17.4 & 18 \\
\hline 13 & 18.8 & 20 \\
\hline 18 & 19.6 & 16 \\
\hline 25 & 23.7 & 8 \\
\hline 27 & 26.5 & No collapse \\
\hline 29 & 28 & 23 \\
\hline 26 & 31.2 & No collapse \\
\hline 17 & 36 & No collapse \\
\hline 16 & 37.6 & No collapse \\
\hline
\end{tabular}

volume $+2 \mathrm{~cm}^{3}$ ) using this value a significant difference, with the Mann-Whitney U test, was still found between the two groups $(p=0.0002)$.

When the percentage of the femoral head affected by osteonecrosis (the volume of the femoral head was assumed to be a sphere) was used as the dependent variable, the difference between the groups with and without collapse on the first MRI evaluation was also significant $(p=0.0000004)$. We estimated that the maximum error in measuring the volume of the femoral head was $8 \mathrm{~cm}^{3}$. Using this value in the worst case, we calculated the percentage of femoral head affected as (volume of osteonecrosis $\left.-2 \mathrm{~cm}^{3}\right) /($ volume of femoral head +8 $\mathrm{cm}^{3}$ ) for the group with osteonecrosis with collapse. In the group with osteonecrosis without collapse, for the most pessimistic simulation, we assumed the percentage of the femoral head affected by the osteonecrosis to be "the volume of osteonecrosis $+2 \mathrm{~cm}^{3}$ " and the volume of the femoral head to be "the volume of the femoral head $-8 \mathrm{~cm}^{3}$ ". When the percentages affected in each group were compared, using the worst case values, the difference between the two groups remained significant $(\mathrm{p}=0.005)$.

In this series, with a maximum follow up of two years (that is, two years after the initial MRI evaluation of size), only seven osteonecroses (among the 60) had not collapsed. For the seven hips without collapse at this time, osteonecrosis was still present on the second MRI. For these seven hips the average volume measured on the second MRI at the last follow up was $0.97 \%$ lower than the volume measured on the first MRI at the time of presentation and 


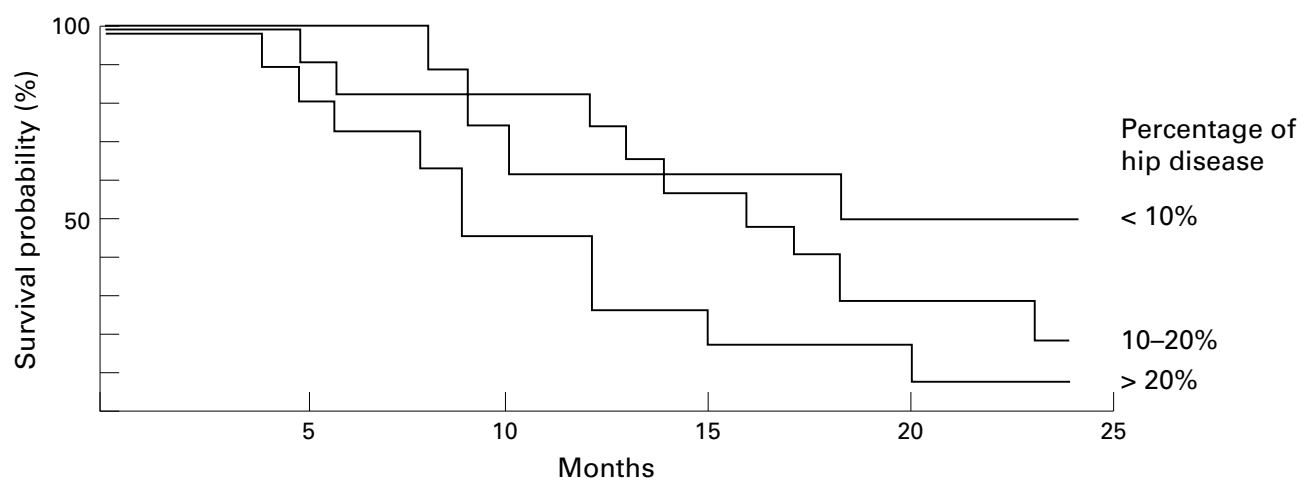

Figure 2 Kaplan-Meier curve showing survival probability before collapse over the two years' follow up for the patients with the femoral head affected to a different degree at presentation.

this difference was not significant. For the 23 hips with collapse at the last follow up and evaluated first at stage I, the average volume measured on MRI after collapse was $1.08 \%$ higher than the volume measured on MRI before collapse; the difference was not significant.

The time between the collapse of the two hips in each patient was related to the significant difference $(\mathrm{p}<0.0000001)$ between the volume of the two osteonecroses calculated on the first MRI of both hips: the time increased as the difference in volume of the two osteonecroses increased (table 2). By logistic regression analysis, the percentage volume of osteonecrosis on the MRI at presention was a significant predictor $(\mathrm{p}<0.01)$ of the time to collapse of the second hip in this group of bilateral osteonecrosis, one side with collapse and the other without. For the selected group of stage I hips, the percentage of the femoral head affected by the necrosis on the first MRI ranged from 0 to $10 \%$ in eight hips and among these eight hips, four $(50 \%)$ had no collapse two years after collapse of the first hip. The percentage of necrosis was greater than $20 \%$ in 11 stage I hips and only one hip had no collapse two years after collapse of the first hip. The difference in survivorship of the stage I hip for the three groups with the femoral head affected to a different degree $(<10 \%, 10-20 \%$, $>20 \%$ ) can be seen in the Kaplan-Meier curve (fig 2).

\section{Discussion}

The goal of this study was to report the rate of progression of osteonecrosis in the asymptomatic hip of patients who were seen owing to collapse and for surgery in the contralateral hip. As many authors have indicated that the extent of the necrotic lesion affects the outcome, ${ }^{14-16}$ we used volumetric analysis of the lesion to investigate the influence of its size on the rate of progression and to determine why the disease progresses more slowly in the asymptomatic hip than in the symptomatic hip. We isolated in this study a subgroup of patients who had received corticosteroid treatment for only a short time, thus allowing determination of the time of onset.

Several authors have used MRI to assess the extent of osteonecrosis with different methods: visual estimation of the percentage of osteo- necrosis in quartiles (Beltran) and volume estimation using $2 \mathrm{~cm}$ slices (Lafforgue). Although these methods are useful and allow indirect volume estimation of osteonecrosis, they are not an exact measure of the volume and do not allow statistical comparisons. The technique we used, well known in mathematics for calculating a volume by the integration of the surface area, is more exact but time consuming. The advantage is that its calculates the "exact" volume and percentage and allows statistical comparisons. Probably, in the next few years, automatic volumetric analysis with 3D software packages will simplify the procedure.

It is well known that patients who have bilateral osteonecrosis of the femoral head may have pain in one or both hips. A clinical dilemma that commonly arises in the assessment of an asymptomatic, radiographically normal hip, but with an abnormal MRI scan at the time that the contralateral hip is evaluated for pain, is to determine the outcome of the asymptomatic diseased femoral head. It is not known how many asymptomatic hips with normal findings on initial radiographs, but with an abnormal MRI scan, will eventually manifest symptoms and radiological changes. The common clinical challenge is the treatment (or not) of such an asymptomatic hip.

In our study 23 of the 30 patients with stage I asymptomatic osteonecrosis of the femoral head progressed to symptomatic disease and to stage III two years after the collapse of the first hip. The unfavourable outcome for most of the asymptomatic hips with an abnormal MRI scan but normal findings on initial radiographs in the present study is in agreement with the findings reported by Bradway and Morrey. ${ }^{17}$ So our experience has led us to believe that most of the hips with MRI positive osteonecrosis of the femoral head will progress to symptomatic disease in patients with a previously collapsed hip. Conservative surgical procedures (before collapse or stage III) are reported to have better results when performed at earlier stages. Thus prediction of the time remaining before collapse in the asymptomatic hip may be important. In this series the volume of the osteonecrosis seemed to predict the radiological progression (occurrence of collapse) of the osteonecrosis of the second hip when patients already had a collapsed hip. The clinical relevance of this observation is that if the 
difference in volume of both osteonecroses is small, when one hip has collapsed, the other asymptomatic hip will probably collapse quickly, at least when the two osteonecroses occurred at the same time or with a short delay. ${ }^{18}$

Several other groups ${ }^{4}$ have begun to use MRI to assess the severity of osteonecrosis. These reports concluded that the size of the lesion had a strong correlation with outcome. $^{8-10}$ Thus our observations with volumetric analysis of the size of the lesion are consistent with a rapidly expanding number of reports. This study was performed in patients with osteonecrosis of both hips. The speed of progression of osteonecrosis varied between the hips and was related to the volume of the osteonecrosis. One of the limits of this study might be the comparison of the volume of osteonecrosis with and without collapse. From a theoretical point of view, the volume of the osteonecrosis is greater before collapse. In this series, however, the average volume measured on MRI after collapse was $1.08 \%$ higher than the volume measured on MRI before collapse. The difference was not significant and may be explained by the fact that after collapse, some mobility of the sequestrum on the underlying normal bone may destroy the repair tissue on the underlying normal bone.

1 Beltran J, Knight CT, Zuelzer WA, Morgan JP, Shwendeman LJ, Chandnani VP, et al. Core decompression for avascular necrosis of the femoral head: correlation between long-term results and preoperative MR staging. Radiology 1990; 175:533-6.

2 Holman A, Gardner G, Richardson M, Simkin P. Quantitative magnetic resonance imaging predicts clinical outcome of care decompression for osteonecrosis of the femoral head. J Rheumatol 1995;22:1929-33.

3 Steinberg ME, Hayken GD, Steinberg DR. A quantitative system for staging avascular necrosis. J Bone Joint Surg B 1995;77:34-41.
4 Schimizu K, Moriya H, Akita T, Sakomoto M, Suguro T. Prediction of collapse with magnetic resonance imaging of avascular necrosis of the femoral head. J Bone Joint Surg Am 1994;76:215-23.

5 Takatory Y, Kokubo T, Ninomia S, Nakamura S, Morimoto S, Kusaba I. Avascular necrosis of the femoral head: natural history and magnetic resonance imaging. J Bone Joint Surg Br 1993;75:217-21.

6 Remington RD, Schork MA. Statistics with applications to the biological and health sciences. Englewood Cliffs, New Jersey: Prentice Hall, 1970

7 Sugano N, Ohzono K, Masuhara K, Takaoka K, Ono K. Prognostication of osteonecrosis of the femoral head in patients with systemic lupus erythematosus by magnetic resonance imaging. Clin Orthop 1995;305:190-9.

$8 \mathrm{Koo} \mathrm{KH}$, Kim R. Quantifying the extent of osteonecrosis of the femoral head: a new method using MRI. J Bone Joint Surg Br 1995;77:825-30.

9 Lafforgue P, Dahan E, Chagnaud C, Schiano A, Kasbarian $\mathrm{M}$, Acquaviva PC. Early stage avascular necrosis of the femoral head: RM imaging for prognosis in 31 cases with at least 2 years of follow-up. Radiology 1993;187:199-204.

10 Le Parc JM, Helenon FB, Paolaggi JB, Kries H. Avascular necrosis of the hip in 305 renal transplant patients. MRI aspects, evolution with and without core decompression after 3 years [abstract]. Arthritis Rheum 1993;36:S170.

11 Bassett LW, Mirra JM, Cracchiolo A, Glod HR. Ischemic necrosis of the femoral head: correlation of magnetic resonance imaging and histologic sections. Clin Orthop 1987;223:181-7.

12 Lang P, Jergensen HE, Moseley ME, Block JE, Chafetz NI, Genant HK. Avascular necrosis of the femoral head: highfield strength MR imaging with histologic correlation. Radiology 1988;169:517-24.

13 Rubin G, Napel S, Leung M. Volumetric analysis of volumetric data: achieving a paradigm shift. Radiology 1996;200:312-17.

14 Jergesen $\mathrm{H}$, Khan $\mathrm{A}$. The natural history of untreated asymptomatic hips in patients who have non traumatic osteonecrosis. J Bone Joint Surg Am 1997;79:359-63.

15 Kopecky KK, Braunstein EM, Brandt KD, Filo RS, Leapman SB, Capello WN, et al. Apparent avascular necrosis of the hip: appearance and spontaneous resolution of MR findings in renal allograft recipients. Radiology 1991;179:523-7.

16 Lee CK, Hansen HT, Weiss AB. The silent hip of idiopathic ischemic necrosis of the femoral head in adults. J Bone Joint Surg Am 1980;62:795-800.

17 Bradway JK, Morrey BF. The natural history of the silent hip in bilateral atraumatic osteonecrosis. J Arthroplasty 1993;8:383-7

18 Sugano N, Nishii T, Shibuya T, Nakata K, Masuhara K, Takaoka K. Contralateral hip in patients with unilateral non traumatic osteonecrosis of the femoral head. Clin Orthop 1997;334:85-90. 